\title{
Modular covariance properties of composite fermions on the torus
}

\author{
Mikael Fremling \\ Institute for Theoretical Physics, Center for Extreme Matter and Emergent Phenomena, Utrecht University, \\ Princetonplein 5, 3584 CC Utrecht, the Netherlands \\ and Department of Theoretical Physics, Maynooth University, Maynooth, Co. Kildare, W23 HW31, Ireland
}

(Received 30 October 2018; published 11 February 2019)

\begin{abstract}
In this work we show that the composite fermion construction for the torus geometry is modular covariant. We show that this is the case both before and after projection and that modular covariance properties are preserved under both exact projection and under JK projection which was recently introduced by $\mathrm{Pu}, \mathrm{Wu}$, and Jain [Phys. Rev. B 96, 195302 (2017)]. It is crucial for the modular properties to hold that the composite fermion state is a proper state, i.e., that there are no vacant $\Lambda$-level orbitals directly underneath any $\Lambda$-level orbital occupied by a composite fermion.
\end{abstract}

DOI: 10.1103/PhysRevB.99.075126

\section{INTRODUCTION}

In the study of the fractional quantum Hall effect, a prominent role has been played by the construction of trial wave functions, dating back to Laughlin's wave function [1] more than three decades ago. The first formulation, which was for a finite quantum liquid on an infinite plane, was subsequently generalized to both a sphere [2] and torus [3]. In the following years other trial wave functions were also constructed on the three geometries, such as the Pfaffian [4-6]. These three geometries: plane, sphere, and torus, have since then been the canonical playground for fractional quantum Hall trial wave functions.

In this work, we build upon and extend recent developments [7] in constructing Jain-Kamilla projected wave functions for composite fermions on the torus geometry. Composite fermions (CF) are straightforward to write down in unprojected form [8-10] on all three above-mentioned geometries. However, to obtain physical wave functions, i.e., that reside in the lowest Landau level (LLL), the CF wave functions need to be projected onto that LLL. This can be achieved either analytically [11] or via the Jain-Kamilla (JK) projection [12]. The former is exact but numerically inefficient, and the latter is an uncontrolled approximation but numerically fast. It was early understood how to perform both of these projections on the plane and sphere, but the torus geometry proved more difficult, mainly due to technical difficulties with the nontrivial interplay of boundary conditions and the action of derivatives on quasiperiodic wave functions. The first successful attempts in this direction was taken by Ref. [10] for the analytical projection.

In a parallel development, trial wave functions on the torus was also developed for the Jain series with the help of CFT [13-15] techniques. Recently DMRG methods have also been extended to the cylinder geometry for the Laughlin state [16], its quasiparticles [17], and states higher up in the hierarchy [18]. See also the construction of quasiparticles for the Laughlin state on the torus [19].

Recently however, $\mathrm{Pu}, \mathrm{Wu}$, and Jain [7] (PWJ) managed to extend the JK-projection scheme to also encompass the torus.
The same techniques were later used in Ref. [20] to study the composite fermion Fermi liquid, which had previously been examined by other numerical techniques [6,21-23].

In comparison to the other two geometries, the torus comes with an extra parameter $\tau$, which controls its geometry. The parameter $\tau$ is important since multiple values of $\tau$ may correspond to the same physical geometry. This redundancy poses additional physical constraints on the trial wave functions that are not present on the plane and sphere, where it is sufficient to respect the boundary conditions. It is therefore of great importance that wave functions defined on the torus not only have correct boundary conditions. One must also require that wave functions at different but physically equivalent values of $\tau$ span the same space. The mapping from one value of $\tau$ to a physically equivalent value is a modular transformation and comes in two flavors: the $T$ transform which sends $\tau \rightarrow \tau+1$ and the $\mathcal{S}$ transform which sends $\tau \rightarrow-\frac{1}{\tau}$. The former is a remapping of the torus lattice vectors, and the latter is a rotation that interchanges the order of the vectors. Sets of wave functions that span the same physical space before and after modular transformations have the property of modular covariance. The modular covariance property was of great importance to compute, e.g., Hall viscosity [14,24-26].

The property of modular covariance is not guaranteed simply because appropriate boundary conditions are imposed. This was made clear in Ref. [14], where it was shown that the primary CFT correlation functions used to construct hierarchy wave functions have the correct modular properties, but that the naive introduction of a regularized derivative (as was previously done in Ref. [13]) broke modular covariance. The authors could find another regularization which restored the modular covariance and as a positive side effect also significantly improved the overlap with the Coulomb ground state.

The property of modular covariance has never been proven for the composite fermion states, neither before nor after projection, and that is what we will do in this paper. On the route towards that, we will also present some useful reformulations and results of the PWJ approach. The paper is organized as 
follows: In Sec. II we introduce the torus geometry and the single particle wave functions. In Sec. III we discuss the Girvin-Jach rule in $\tau$ gauge. In Sec. IV we briefly discuss the CF construction on the torus and in Sec. V we discuss the modification of the Grivin-Jach rule that is necessary to obtain periodic boundary conditions for the Jain-Kamilla projection. In Sec. VI we derive the covariance properties for unprotected as well as exactly projected and PWJ projected CFs and show that they all satisfy modular covariance. We end with a discussion and outlook in Sec. VII. Detailed derivations are deferred to the appendices.

\section{THE TORUS AND ITS WAVE FUNCTIONS}

In this section we give a short recapitulation regarding the torus. This also serves to define the notation that is used later in the paper. The torus is defined by two axes $\boldsymbol{L}_{1}, \boldsymbol{L}_{2}$ on the plane and we will adopt the conventions that in complex coordinates the axes are $L_{1}=L$ and $L_{2}=\tau L$, where $\tau=$ $\tau_{1}+\imath \tau_{2}$. For coordinates we use the convention that $z=\tilde{x}+$ $\imath \tilde{y}=L(x+\tau y)$, where $\tilde{x}, \tilde{y}$ are the euclidean (dimensional) coordinates and $x, y$ are the reduced (dimensionless) coordinates. The reduced coordinates $x, y \in[0,1]$, defined on the unit square, are convenient since $x=1(y=1)$ corresponds to $z=L_{1}\left(z=L_{2}\right)$. The two torus axes span an area $\left|\boldsymbol{L}_{1} \times \boldsymbol{L}_{2}\right|=$ $\tau_{2} L^{2}=2 \pi N_{\phi} \ell_{B}^{2}$ where $\ell_{B}=\sqrt{\frac{\hbar e}{B}}$ is the magnetic length and $N_{\phi}$ is the number of magnetic fluxes that penetrate its surface. See Fig. 1 for an illustration of the coordinates and gauge choice.

The single particle Hamiltonian is

$$
H=\sum_{i=\tilde{x}, \tilde{y}} \frac{1}{2 m}\left(p_{j}-e \tilde{A}_{j}\right)^{2},
$$

where $p_{j}=\imath \hbar \partial_{j}$ and $\overrightarrow{\tilde{A}}=\sum_{i=\tilde{x}, \tilde{y}} \tilde{A}_{i} \hat{i}$ is a vector potential satisfying $\nabla \times \overrightarrow{\tilde{A}}=B \hat{z}$. We will choose to work in the $\tau$ gauge, where the vector potential is $\overrightarrow{\tilde{A}}=\frac{\tilde{y} B}{\tau_{2}}\left(\tau_{2},-\tau_{1}\right)$, which is perpendicular to the vector $\vec{\tau}$. In reduced coordinates the vector potential simplifies to $\vec{A}=\left(2 \pi N_{\phi} y B, 0\right)$ which is explicitly $\tau$ independent. In this work we will work exclusively in $\tau$ gauge, as it is especially convenient to handle modular

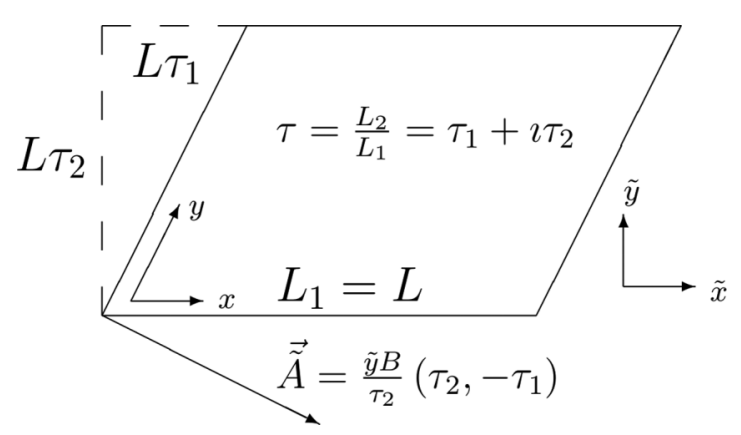

FIG. 1. The relationship between the Cartesian coordinates $(\tilde{x}, \tilde{y})$ and the dimensionless coordinates $(x, y)$. In the figure one can also see that $\tau_{1}$ is interpreted as the skewness, and $\tau_{2}$ as the aspect ratio, of the torus. Note how the $\tau$-gauge vector potential $\overrightarrow{\tilde{A}}$ is perpendicular to the vector $\vec{\tau}=\left(\tau_{1}, \tau_{2}\right)$. The area of the torus is fixed to be $L^{2} \tau_{2}=$ $2 \pi N_{\phi} \ell_{B}$. transformations and boundary conditions at arbitrary $\tau$. The Hamiltonian in (1) can be diagonalized by introducing ladder operators, yielding the form $H=\hbar \omega_{B}\left(a^{\dagger} a+\frac{1}{2}\right)$, where $\omega_{B}=$ $\frac{e B}{m}$. The ladder operators in $\tau$ gauge are

$$
\begin{aligned}
& a_{\tau}=\sqrt{2}\left(\frac{\tau}{2} \frac{L}{\ell_{B}} y+\ell_{B} \partial_{\bar{z}}\right) \\
& a_{\tau}^{\dagger}=\sqrt{2}\left(\frac{\bar{\tau}}{2} \frac{L}{\ell_{B}} y-\ell_{B} \partial_{z}\right),
\end{aligned}
$$

and satisfy $\left[a_{\tau}^{\dagger}, a_{\tau}\right]=1$. Physical wave functions $\psi(z)$ are quasiperiodic and obey the boundary conditions

$$
\psi\left(z+L_{j}\right)=e^{\lambda_{j}(z, \bar{z})} \psi(z)
$$

where $\lambda_{j}(z, \bar{z})$ depends on the gauge choice $\vec{A}$. For $\tau$ gauge this is $\lambda_{j}=\delta_{j, 2} 2 \pi N_{\phi} x$.

In $\tau$ gauge, the shift operator and magnetic translation operators are

$$
\begin{aligned}
& \tilde{t}(\alpha L+\beta \tau L)=e^{\alpha \partial_{x}+\beta \partial_{y}} \\
& t(\alpha L+\beta \tau L)=e^{\alpha \partial_{x}+\beta \partial_{y}+t 2 \pi \beta N_{\phi} x},
\end{aligned}
$$

where it is the latter that defines periodic boundary conditions $t\left(L_{j}\right) \psi=e^{\imath \phi_{j}} \psi$. Note how we differentiate between the shift operator $\tilde{t}(\tau L)$ and the full magnetic translation operator $t(\tau L)$ such that $t(\tau L)=e^{i 2 \pi N_{\phi} x} \tilde{t}(\tau L)$. General LLL wave functions in $\tau$ gauge take the form $\psi^{(A)}(z)=e^{i 2 \pi A y^{2}} f(z)$ where $f(z)$ is a holomorphic function and $A$ counts the number of magnetic fluxes through the torus. The above formula is particularity useful, since if $\psi^{(A)}$ and $\psi^{(B)}$ are wave functions with boundary conditions $\phi_{A}$ and $\phi_{B}$ then $\psi^{(A+B)}=\psi^{(A)} \cdot \psi^{(B)}$ is automatically a wave function with boundary conditions $\phi_{A}+\phi_{B}$.

The operator $t\left(\frac{L}{N_{\phi}}\right)$ commutes with the operator $t(\tau L)$ and consequently can be used to define a basis of $N_{\phi}$ linearly independent states. The single particle orbitals in the lowest Landau level—in a basis that diagonalizes $t\left(\frac{L}{N_{\phi}}\right)$ - can be written as

$$
\begin{aligned}
\phi_{i}^{\left(N_{\phi}\right)} & =\frac{1}{\sqrt{\ell_{B} L \sqrt{\pi}}} e^{l \pi \tau N_{\phi} y^{2}} \vartheta_{\frac{i}{N_{\phi}}, 0}\left(\frac{N_{\phi} z}{L} \mid N_{\phi} \tau\right) \\
& =\frac{1}{\sqrt{\ell_{B} L \sqrt{\pi}}} \sum_{k \in \mathbb{Z}+\frac{i}{N_{\phi}}} e^{\imath \pi N_{\phi} \tau(y+k)^{2}} e^{l 2 \pi N_{\phi} k x} .
\end{aligned}
$$

The function $\vartheta_{a, b}(z \mid \tau)=\sum_{k \in \mathbb{Z}+a} e^{l \pi \tau k^{2}} e^{l 2 \pi k(z+b)}$ is a generalized Jacobi $\theta$ function. The orbitals for the higher Landau levels are obtained by application of the raising operators as $\phi_{j, n}=\frac{\left(a^{\dagger}\right)^{n}}{\sqrt{n !}} \phi_{j}$. The explicit expression for the $n$th Landau level orbitals, also as eigenstates of $t\left(\frac{L}{N_{\phi}}\right)$, are

$$
\begin{aligned}
\phi_{i, n}^{\left(N_{\phi}\right)} & =\mathcal{N}_{n} \sum_{k \in \mathbb{Z}+\frac{i}{N_{\phi}}} e^{\imath \pi N_{\phi} \tau(y+k)^{2}} e^{i 2 \pi N_{\phi} k x} H_{n}\left(\frac{\tau_{2} L}{\ell_{B}^{2}}(y+k)\right) \\
& =\mathcal{N}_{n} e^{\imath \pi N_{\phi} \tau y^{2}} \sum_{k \in \mathbb{Z}+\frac{i}{N_{\phi}}} e^{\imath \pi N_{\phi} \tau k^{2}} e^{l 2 \pi N_{\phi} k \frac{z}{L}} H_{n}\left(\tilde{y}+\tau_{2} L k\right),
\end{aligned}
$$

where $H_{n}$ is a Hermite polynomial and $\mathcal{N}_{n}=\frac{1}{\sqrt{2^{n} n ! L \sqrt{\pi}}}$. Note the appearance of the physical $\tilde{y}=L \tau y$ in the argument of the 
Hermite polynomial. We will refer to $f_{i, n}^{\left(N_{\phi}\right)}$ as the holomorphic polynomial in $\phi_{i, n}^{\left(N_{\phi}\right)}=e^{\imath \pi N_{\phi} \tau y^{2}} f_{i, n}^{\left(N_{\phi}\right)}$, and we will often drop the momentum index $i$ for brevity.

Haldane has in recent papers advocated for the use of Weierstrass $\sigma$ functions $[21,23,27,28]$ over the traditionally used $\vartheta$ functions. In this paper we follow in that tradition and define a generalized $\sigma$ function in $\tau$ gauge as

$$
\sigma_{a, b}^{(n)}(z)=e^{\imath \pi \tau n y^{2}} \vartheta_{a, b}\left(\frac{n z}{L} \mid n \tau\right) .
$$

Comparing with (5) we have, e.g., that $\sigma_{\frac{i}{N_{\phi}}, 0}^{\left(N_{\phi}\right)}=$ $\sqrt{\ell_{B} L \sqrt{\pi}} \phi_{i}^{\left(N_{\phi}\right)}$. The Weierstrass $\sigma$ functions build in the quasiperiod boundary conditions and thus transform under coordinate changes as

$$
\begin{aligned}
\sigma_{a, b}^{(n)}(z+L) & =e^{l 2 \pi a n} \sigma_{a, b}^{(n)}(z) \\
\sigma_{a, b}^{(n)}(z+L \tau) & =e^{-l 2 \pi(n x+b)} \sigma_{a, b}^{(n)}(z),
\end{aligned}
$$

in accordance with (3). With this definition, one may also rewrite the $q$-fold degenerate Laughlin state on the torus, which in $\vartheta$ form is

$$
\begin{aligned}
\psi_{\frac{1}{q}}= & \mathcal{N}(\tau) e^{l \pi \tau N_{\phi} \sum_{i} y_{i}^{2}} \vartheta_{0,0}\left(\frac{q \sum z_{i}}{L} \mid q \tau\right) \\
& \times \prod_{i<j} \vartheta_{\frac{1}{2}, \frac{1}{2}}\left(\frac{z_{i}-z_{j}}{L} \mid \tau\right)^{q} .
\end{aligned}
$$

In Weierstrass form this is the more compact

$$
\psi_{\frac{1}{q}}=\mathcal{N}(\tau) \sigma_{0,0}^{(q)}\left(\sum_{i} z_{i}\right) \prod_{i<j} \sigma_{\frac{1}{2}, \frac{1}{2}}^{(1)}\left(z_{i}-z_{j}\right)^{q} .
$$

The normalization factor is here chosen to be $\mathcal{N}(\tau)=$ $\frac{\left[\sqrt{\tau_{2}} \eta^{2}(\tau)\right]^{\frac{q N_{e}}{2}}}{\eta(\tau)^{\frac{q N_{e}\left(N_{e}-1\right)}{2}}+1}$ as suggested in Ref. [24], where $\eta(\tau)=$ $e^{\frac{i \pi \tau}{12}} \prod_{n=1}^{\infty}\left(1-e^{2 \pi \imath n \tau}\right)$ is the Dedekind $\eta$ function. This normalization ensures that the Laughlin state transforms under $\mathcal{S}$ transformations as $\psi_{\frac{1}{q}} \rightarrow\left(\frac{\tau}{|\tau|}\right)^{\frac{N_{e q}}{2}} \psi_{\frac{1}{q}}$ and is the correct one (up to $\tau$-independent scale factors) as long as the torus is large enough [29].

\section{GIRVIN-JACH PROJECTION IN $\tau$ GAUGE}

In their work in Ref. [11], Girvin and Jach introduced the classic rule for LLL projection, namely that $\bar{z} \rightarrow 2 \partial_{z}$. What might not be obvious is that this is a gauge dependent rule and is only guaranteed to hold in symmetric gauge. In this section we review the Girvin-Jach projection trick [11] and then extend it to $\tau$ gauge. We begin by reminding ourselves of the argument that goes in symmetric gauge, before we turn to the $\tau$ gauge. The ladder operators in symmetric gauge are

$$
\begin{aligned}
& a_{s}=\sqrt{2}\left(\frac{z}{4 \ell_{B}}+\ell_{B} \partial_{\bar{z}}\right) \\
& a_{s}^{\dagger}=\sqrt{2}\left(\frac{\bar{z}}{4 \ell_{B}}-\ell_{B} \partial_{z}\right),
\end{aligned}
$$

where the $s$ denotes the symmetric gauge choice. The equation for $a^{\dagger}$ can be rewritten as $\bar{z}=\frac{4 a_{s}^{\dagger}}{\sqrt{2}}+4 \partial_{z}$ (where we have dropped factors of $\left.\ell_{B}\right)$. This allows us to write

$$
\begin{aligned}
\bar{z} e^{-\frac{z \bar{z}}{4}} f(z) & =\left(\frac{4 a_{s}^{\dagger}}{\sqrt{2}}+4 \partial_{z}\right) e^{-\frac{z \bar{z}}{4}} f(z) \\
& =\frac{4 a_{s}^{\dagger}}{\sqrt{2}} e^{-\frac{z \bar{z}}{4}} f(z)+e^{-\frac{z \bar{z}}{4}}\left(4 \partial_{z}-\bar{z}\right) f(z),
\end{aligned}
$$

or equivalently

$$
\bar{z} e^{-\frac{z \bar{z}}{4}} f(z)=\sqrt{2} a_{s}^{\dagger} e^{-\frac{z \bar{z}}{4}} f(z)+2 e^{-\frac{z \bar{z}}{4}} \partial_{z} f(z) .
$$

Applying the LLL projection kills the $a^{\dagger}$ term and we have $P_{\mathrm{LLL}} \bar{z} e^{-\frac{z \bar{z}}{4}} f(z)=2 e^{-\frac{z \bar{z}}{4}} \partial_{z} f(z)$ which amounts to the famous rule [11] $\bar{z} \rightarrow 2 \partial_{z}$, where it is understood that the derivative does not act on the exponential $\boldsymbol{e}^{-\frac{z \bar{z}}{4}}$. Here, since $\left[a_{s}^{\dagger}, \bar{z}\right]=0$ the argument can also be iterated to higher powers of $\bar{z}$ as $\bar{z}^{n} \rightarrow\left(2 \partial_{z}\right)^{n}$. In $\tau$ gauge, due to (2), the same equation reads $\frac{\bar{\tau}}{2} L y=\frac{a_{\tau}^{\bar{\tau}}}{\sqrt{2}}+\partial_{z}$, which becomes the equation

$$
\frac{\bar{\tau}}{2} L y G_{\tau} f(z)=\frac{a_{\tau}^{\dagger}}{\sqrt{2}} G_{\tau} f(z)+G_{\tau}\left(\partial_{z}+\frac{L \tau y}{2}\right) f(z)
$$

after acting on $G_{\tau}=e^{\imath \pi \tau N_{\phi} y^{2}}$. The above equation may be rewritten as

$$
\tau_{2} L y G_{\tau} f(z)=\frac{a_{\tau}^{\dagger} l}{\sqrt{2}} G_{\tau} f(z)+G_{\tau}\left(l \partial_{z}\right) f(z) .
$$

After projection (and the $a_{\tau}^{\dagger}$ term is killed) this becomes $\tilde{y}=$ $\tau_{2} L y \rightarrow \imath \partial_{z}$, with the understanding that $\partial_{z}$ does not act on the Gaussian factor $e^{\imath \tau \tau N_{\phi} y^{2}}$. The rule for $\tilde{y}$ can however not be extended directly to higher powers of $\tilde{y}$ since $\left[\tilde{y}, a_{\tau}^{\dagger}\right] \neq 0$. Instead, due to this noncommutativity the projection rule reads

$$
\tilde{y}^{n} \rightarrow \frac{1}{(-2 \iota)^{n}} H_{n}\left(\partial_{z}\right),
$$

where $H_{n}$ is a Hermite polynomial. A proof and an extended discussion can be found in Appendix A. We wish to stress that since the $P_{\mathrm{LLL}}$ operator only involves $a$ and $a^{\dagger}$ operators that act between LL, it trivially commutes with the operators within any LLL. This has the important consequence that if a wave function satisfies the boundary conditions before projection, it will automatically do so also after projection.

\section{A. LLL projection as an operator}

Here we develop a formalism where we view the LLL projection as an operator action on holomorphic LLL wave functions. To be concrete, we consider a general state (e.g., basis state) $\phi_{n}^{(M)}$ in the $n$th LL defined for $M$ fluxes, that is multiplied with an arbitrary LLL wave function $\psi^{\left(N_{\phi}-M\right)}$ defined for $N_{\phi}-M$ fluxes. The power of the $\tau$-gauge formalism and reduced coordinates is that the product of $\phi_{n}^{(M)} \psi^{\left(N_{\phi}-M\right)}$ (when expressed in reduced coordinates) is automatically a proper wave function at $N_{\phi}$ fluxes, since the different magnetic lengths $\ell_{B}$ of the two wave functions are automatically renormalized.

The product $\phi_{n}^{(M)} \psi^{\left(N_{\phi}-M\right)}$ can now be written as

$$
\phi_{n}^{(M)} \psi^{\left(N_{\phi}-M\right)}=e^{\imath \pi \tau N_{\phi} y^{2}} f_{n}^{(M)} f^{\left(N_{\phi}-M\right)},
$$

where $\psi^{\left(N_{\phi}-M\right)}=e^{\imath \pi \tau\left(N_{\phi}-M\right) y^{2}} f^{\left(N_{\phi}-M\right)}$ is separated into its Gaussian and holomorphic factor, and the same for $\phi_{n}^{(M)}=$ 
$e^{\imath \pi \tau M y^{2}} f_{n}^{(M)}$. When applying $P_{\text {LLL }}$ on this combined wave function we can use the fact that only $\phi_{n}^{(M)}$ is nonholomorphic and promote $f_{n}^{(M)}$ to a differential operator acting on $f^{\left(N_{\phi}-M\right)}$ as

$$
P_{\mathrm{LLL}} \phi_{n}^{(M)} \psi^{\left(N_{\phi}-M\right)}=e^{\imath \pi \tau N_{\phi} y^{2}} \hat{f}_{n}^{(M)} f^{\left(N_{\phi}-M\right)} .
$$

The operator $\hat{f}_{n}^{(M)}$ can after some transformations (see Appendix B) be rewritten as

$$
\hat{f}_{n}^{(M)}=\sum_{k=0}^{n}\left(\begin{array}{l}
n \\
k
\end{array}\right)\left(M \partial_{z}\right)^{n-k}\left[\left(-N_{\phi} \partial_{z}\right)^{k} f_{0}\right],
$$

where $f_{0}=e^{-\imath \pi M y^{2}} \frac{a^{n}}{\sqrt{n !}} \phi_{n}$ is the LLL version of $f_{n}$ and where a scale factor of $(2 l)^{n} \frac{\mathcal{N}_{n}}{\mathcal{N}_{0} M^{n}}$ has been suppressed [30]. The derivative within square brackets acts only on $f_{0}$.

We may symbolically write the operator in (11) as

$$
\hat{f}_{n}^{(M)}=\left(M \partial_{z}-N_{\phi} \tilde{\partial}_{z}\right)^{n} f_{0},
$$

where the operator $\tilde{\partial}_{z}$ is understood to act only on $f_{0}$ and thus has the property $\tilde{\partial}_{z} f_{0} f=f \tilde{\partial}_{z} f_{0}$. We may also introduce the derivative operator $\hat{\partial}_{z}$ which does not act on $f_{0}$ at all and can be defined as $\hat{\partial}_{z} f_{0} f=f_{0} \hat{\partial}_{z} f$. Using that these two operators have the identity $\partial_{z}=\tilde{\partial}_{z}+\hat{\partial}_{z}$ and that the three operators $\partial_{z}$, $\tilde{\partial}_{z}, \hat{\partial}_{z}$ all commute, we may rewrite (12) as

$$
\hat{f}_{n}=\left(N_{\phi} \hat{\partial}_{z}-\left(N_{\phi}-M\right) \partial_{z}\right)^{n} f_{0},
$$

and

$$
\hat{f}_{n}=\left(M \hat{\partial}_{z}-\left(N_{\phi}-M\right) \tilde{\partial}_{z}\right)^{n} f_{0},
$$

where especially (14) will be useful later. This is also the form that was found by PWJ. For brevity we will also introduce the operator $\hat{D}=M \hat{\partial}_{z}-\left(N_{\phi}-M\right) \tilde{\partial}_{z}$ such that (14) can be written in shorthand as $\hat{f}_{n}=\hat{D}^{n} f_{0}$.

\section{B. Periodic boundary conditions of $\hat{f}_{n}$}

To set the stage for the discussion of the PWJ projection in the later sections we now prove that $\hat{f}_{n}$ indeed provides for periodic boundary conditions. We know that an $A$ flux wave function $\psi^{(A)}=e^{l \pi \tau A y^{2}} f^{(A)}$ should obey the relation $t(\tau L) \psi^{(A)}=\psi^{(A)}$ (assuming p.b.c). Removing the factor $e^{l \pi \tau A y^{2}}$ and the gauge factor $e^{l 2 \pi A x}$ we see that this implies that $\tilde{t}(\tau L) f^{(A)}=e^{-l 2 \pi A\left(z+\frac{\tau}{2}\right)} f^{(A)} \tilde{t}(\tau L)$. This means that the relation

$$
\left(\tilde{t}(\tau L) \hat{f}_{n}\right) e^{-l 2 \pi\left(N_{\phi}-M\right)\left(z+\frac{\tau}{2}\right)}=e^{-l 2 \pi N_{\phi}\left(z+\frac{\tau}{2}\right)} \hat{f}_{n}
$$

should hold for $\hat{f}_{n}$. Note here that $[\tilde{t}(\tau L), \hat{D}]=0$, but that $\tilde{t}(\tau L) f_{0}=e^{-l 2 \pi M\left(z+\frac{\tau}{2}\right)} f_{0} \tilde{t}(\tau L)$. This means that when $\tilde{t}(\tau L)$ acts on $f_{0}$ it will produce the factor $e^{-l 2 \pi M\left(z+\frac{\tau}{2}\right)}$. This factor will be acted upon by $\tilde{\partial}_{z}$ in Eq. (14), effectively causing the shift $\tilde{\partial}_{z} \rightarrow \tilde{\partial}_{z}-\imath 2 \pi M$. Likewise when $\tilde{t}(\tau L)$ acts on $f^{\left(N_{\phi}-M\right)}$ it produces the factor $e^{-\imath 2 \pi\left(N_{\phi}-M\right)\left(z+\frac{\tau}{2}\right)}$, which when pulled through $\hat{\partial}_{z}$ causes the shift $\hat{\partial}_{z} \rightarrow \hat{\partial}_{z}-\imath 2 \pi\left(N_{\phi}-M\right)$. Since the two shifts are simple constant they commute and we have

$$
\begin{aligned}
\hat{D}= & M \hat{\partial}_{z}-\left(N_{\phi}-M\right) \tilde{\partial}_{z} \\
& \rightarrow M\left(\hat{\partial}_{z}-\imath 2 \pi\left(N_{\phi}-M\right)\right) \\
& -\left(N_{\phi}-M\right)\left(\tilde{\partial}_{z}-\imath 2 \pi M\right)=\hat{D},
\end{aligned}
$$

when the exponentials are pulled through $\hat{D}$. This shows that $\hat{D}$ is invariant and proves (15).

We mention in passing that we may define $\hat{\phi}_{n}^{(M)}=$ $e^{\imath \tau \tau N_{\phi} y^{2}} \hat{f}_{n}^{(M)} e^{-l \pi \tau\left(N_{\phi}-M\right) y^{2}}$ which is an operator that has proper operator boundary conditions. This operator may be expressed as

$$
\hat{\phi}_{n}=\sum_{k=0}^{\left\lceil\frac{n}{2}\right\rceil} T(n, k) \chi^{k} D^{n-2 k} \phi_{0},
$$

where $T(k, n)$ is the triangle of Bessel numbers [31] and $\chi=$ $\frac{M\left(N_{\phi}-M\right) \tau}{4 l \tau}$. This has been confirmed by Mathematica up to $n=$ 8 , and we assume it holds for general $n$. See Appendix C for details.

\section{COMPOSITE FERMIONS ON THE TORUS}

In this section we briefly introduce the $\mathrm{CF}$ construction on the torus at filling fraction $v=\frac{n}{2 n p+1}$ and discuss how the expected degeneracy of $q=2 p n+1$ comes about. A generic $\mathrm{CF}$ wave function may be written on the form

$$
\psi_{\mathrm{CF}}=P_{\mathrm{LLL}} \chi_{n} \psi_{v=\frac{1}{2 p}},
$$

where $\chi_{n}$ is a Slater determinant of occupied CF orbitals given by (6), where the CF flux is $M$. If $\psi_{\mathrm{CF}}$ represents a ground state at filling fraction $v=\frac{n}{2 p n+1}$, then $n M=N_{e}$ and $N_{\phi}=M+2 p N_{e}$, meaning that the $n$ lowest CF $\Lambda$ levels are filled. As $\psi_{\nu=\frac{1}{2 p}}$ contains a center of mass piece and a Jastrow factor [see Eq. (8)] we may pull the Jastrow factor into the determinant and write

$$
\psi_{\mathrm{CF}}=P_{\mathrm{LLL}} \sigma_{a}^{(2 p)}(Z) \cdot \mathcal{A}\left\{\prod_{j} \phi_{j}\left(z_{j}\right) \cdot J_{j}^{p}(z)\right\} .
$$

Here, $\mathcal{A}$ is an antisymmetrizer of the coordinates that plays the same role as the determinant, and $J_{j}(z)=\prod_{k \neq j} \sigma_{\frac{1}{2}, \frac{1}{2}}^{(1)}\left(z_{j k}\right)$. The subscript $a$ on $\sigma_{a}^{(2 p)}(Z)$ is labeling one of the $2 p$ states of $\psi_{v=\frac{1}{2 p}}$ and the subscript $j$ on $\phi_{j}$ contains for brevity both the LL index and the orbital index. We will later see that it is crucial for the PWJ projection recipe that the CF state is a proper state. A proper CF state has the property that there are no vacant $\Lambda$-level orbitals directly underneath any $\Lambda$-level orbital occupied by a composite fermion. In other words, if the orbital $\phi_{j, n}$ is occupied (with $n>0$ ), then the orbital $\phi_{j, n-1}$ is also occupied.

\section{Notes on the multiplicity of the wave functions}

Here we mention for completeness how the correct degeneracy of the CF states is counted. It is well known that for a LL with partial filling $v=\frac{p}{q}$ ( $p, q$ being relatively prime) every state is at least $q$-fold degenerate on the torus [32] (with higher degeneracy for non-Abelian states). To show this degeneracy explicitly for the CF states, we make use of the many-body translation operator commutations relations $T^{(A)}(\alpha \tau L) t^{(A)}(L)=e^{i 2 \pi A \alpha}$, where the $(A)$ denotes that the wave functions act on $A$-flux wave functions. The many body 
operators are

$$
T^{(A)}(\gamma)=\prod_{j=1}^{N_{e}} t_{j}^{(A)}(\gamma),
$$

where $t_{j}^{(A)}(\gamma)$ is the magnetic translation operator in (4) acting on coordinate $j$. We next define the translated state $\psi_{\mathrm{CF}}^{(\alpha)}=$ $T^{\left(N_{\phi}\right)}(\alpha \tau L) \psi_{\mathrm{CF}}$. If we assume that $\psi_{\mathrm{CF}}$ has periodic boundary conditions then $\psi_{\mathrm{CF}}^{(\alpha)}$ will also have periodic boundary conditions when $e^{i 2 \pi N_{\phi} \alpha}=1$, which happens first when $\alpha=$ $\frac{1}{N_{\phi}}=\frac{n}{N_{e}(2 p n+1)}$. Naively one might expect that there should be $\frac{1}{\alpha}=N_{\phi}$ degenerate states from this argument, which is clearly wrong. To get the correct counting, one has to also take into account that the trivial cycle (i.e., the cycle that sends $\psi_{\mathrm{CF}}^{(\alpha)}=$ $\left.\psi_{\mathrm{CF}}^{(0)}\right)$ is not $\alpha=1$, but is determined by the trivial cycles of $\psi_{\nu=1}^{2 p}$ and $\chi_{n}$. The trivial cycle for $\psi_{\nu=1}$ is $\alpha^{\prime}=\frac{1}{N_{e}}$ since that cycles the states $\phi_{j, 0}^{\left(N_{e}\right)} \rightarrow \phi_{j+1,0}^{\left(N_{e}\right)}$ leaving the $\psi_{\nu=1}$ invariant. In a similar manner, the trivial cycle for $\chi_{n}$ is $\alpha^{\prime \prime}=\frac{1}{M}=\frac{n}{N_{e}}$ since it sends $\phi_{j, k}^{(M)} \rightarrow \phi_{j+1, k}^{(M)}$ in the determinant. We thus see that $(2 p n+1) \alpha=n \alpha^{\prime}=\alpha^{\prime \prime}$ which shows that $2 p n+1=q$ applications of $\alpha$ are needed to obtain trivial cycles for the two subfactors. This shows that the degeneracy of $\psi_{\mathrm{CF}}$ is $q=2 p n+1$ as expected.

\section{MODIFIED JK PROJECTION}

We now discuss the modification to (14) that is necessary to obtain JK-projected wave functions that respect the periodic boundary conditions. In a naive implementation of the JK projection that we would move the projector into the determinant and perform the LLL projection on each term of the determinant. On the plane and sphere this is an uncomplicated procedure, but of the torus this is highly nontrivial since the boundary conditions of the factor $J_{j}(z)$ depends on the other $k \neq j$ coordinates. Nevertheless, we may be bold and stipulate that we can still use (13) and then hope for the best. In that case we first extract the Gaussian factors and write

$$
\begin{aligned}
\psi_{\mathrm{JK}}= & \sigma_{a}^{(2 p)}(Z) e^{\imath \pi \tau\left(N_{\phi} \sum_{j} y_{j}^{2}-2 p Y^{2}\right)} \\
& \times \mathcal{A}\left\{\prod_{j} \hat{f}_{j} \cdot F_{j}^{p}(z)\right\},
\end{aligned}
$$

where now $\hat{f}_{j}$ only acts on the function

$$
F_{j}^{p}(z)=J_{j}^{p} e^{-\imath \pi \tau p \sum_{k \neq j}\left(y_{j}-y_{k}\right)^{2}} .
$$

Here, and below, we use the abbreviations $Y=\sum_{j} y_{j}, X=$ $\sum_{j} x_{j}$ and $Y_{j}=Y-y_{j}=\sum_{k \neq j} y_{k}, X_{j}=X-x_{j}=\sum_{k \neq j} x_{k}$. Here the number of fluxes in $\hat{f}_{j} \cdot F_{j}^{p}(z)$ that the $z$ coordinates sees is $N_{\phi}=M+p\left(N_{e}-1\right)$, instead of $N_{\phi}=M+2 p N_{e}$. To see why this does not work, and also determine what does, we follow the reasoning of PWJ and introduce a modification of $\hat{f}_{j}$ that is $\tilde{f}_{n}=\tilde{D}^{n} f_{0}$, where

$$
\tilde{D}=\alpha M \hat{\partial}_{z}-\left(N_{\phi}-M\right) \tilde{\partial}_{z}=\alpha M \hat{\partial}_{z}-2 p N_{e} \tilde{\partial}_{z} .
$$

For $\alpha=1$ then $\tilde{D}=\hat{D}$ and $\hat{f}_{j}=\tilde{f}_{j}$, but we will soon see that the choice $\alpha=2$ will be necessary. We begin with reviewing the relevant transformations. Acting with $\tilde{t}_{j}(\tau L)$ on $F_{l}$ produces, after we have dropped some constant factors

$$
\begin{aligned}
\tilde{t}_{j}(\tau L) F_{j}(z) & \propto e^{-\imath 2 \pi \prod_{k \neq j}\left(z_{j}-z_{k}\right)} \prod_{k \neq j} \vartheta_{1}\left(z_{j}-z_{k} \mid \tau\right) \tilde{t}_{j}(\tau L) \\
& =e^{-\imath 2 \pi\left[\left(N_{e}-1\right) z_{j}-Z_{j}\right]} F_{j} \tilde{t}_{j}(\tau L)
\end{aligned}
$$

and

$$
\begin{aligned}
\tilde{t}_{j}(\tau L) F_{l \neq j}(z) & \propto e^{-l 2 \pi\left(z_{l}-z_{j}\right)} \prod_{k \neq l} \vartheta_{1}\left(z_{l}-z_{k} \mid \tau\right) \tilde{t}_{j}(\tau L) \\
& =e^{-l 2 \pi\left(z_{l}-z_{j}\right)} F_{l \neq j} \tilde{t}_{j}(\tau L),
\end{aligned}
$$

depending on if $j=l$ or not. We now apply the translation operator $\tilde{t}_{j}(\tau L)$ on $\tilde{f}_{j} F_{j}$. For brevity we suppress the factors of $e^{-l 2 \pi\left[\left(N_{e}-1\right) z_{j}-Z_{j}\right]}$ and $e^{-l 2 \pi\left(z_{l}-z_{j}\right)}$ coming from (22) and (23) as well as the phase $e^{t 2 \pi z_{j} M}$ coming from $f_{0}^{(M)}$. We obtain, by an analogous calculation to the one in (16), that

$$
\begin{aligned}
\hat{f}_{n}^{(M)} F_{j}^{p} \rightarrow & \left(-2 N_{e} p\left(\tilde{\partial}_{z_{j}}-\imath 2 \pi M\right)\right. \\
& \left.+\alpha M\left(\hat{\partial}_{z_{j}}-\imath 2 \pi p\left(N_{e}-1\right)\right)\right)^{n} f_{0} F_{j}^{p} \\
= & \left(\tilde{D}+\imath 2 \pi p M\left[2 N_{e}-\alpha\left(N_{e}-1\right)\right]\right)^{n} f_{0} F_{j}^{p},
\end{aligned}
$$

for $j=l$. For $j \neq l$ we instead have

$$
\begin{aligned}
\hat{f}_{n}^{(M)} F_{l \neq j} & \rightarrow\left(-2 N_{e} p \tilde{\partial}_{z_{j}}+\alpha M\left(\hat{\partial}_{z_{j}}+\imath 2 \pi p\right)\right)^{n} f_{0} F_{l}^{p} \\
& =(\tilde{D}+\alpha p M \imath 2 \pi)^{n} f_{0} F_{l}^{p} .
\end{aligned}
$$

The important observation here is that both $\alpha p M \imath 2 \pi$ and $\imath 2 \pi p M\left[2 N_{e}-\alpha\left(N_{e}-1\right)\right]$ are constants, but they are only equal when $\alpha=2$. It is crucial that the transformation $\tilde{D}^{n} \rightarrow$ $(\tilde{D}+\text { const })^{n}$ is the same for all coordinates, since the shift $\imath \pi 4 p M$ can then be removed by row addition if the CF state is a proper state. Otherwise the cancellation will not work.

As a minimal example let us consider the simple case of a determinant consisting of only $N_{e}=2$ particles; one in the $n=0 \mathrm{LL}$ and one in the $n=1 \mathrm{LL}$. The entries in (19) are then $D f F$ and $f F$, which gives determinant

$$
\left|\begin{array}{cc}
D_{1} f_{1} F_{1} & D_{2} f_{2} F_{2} \\
f_{1} F_{1} & f_{2} F_{2}
\end{array}\right|,
$$

where the subscripts labels the coordinates of the two particles. If we assume that $D_{1} \rightarrow D_{1}+\alpha$ and $D_{2} \rightarrow D_{2}+\beta$ under the action of $t_{1}(\tau L)$, we then have

$$
\begin{aligned}
\left|\begin{array}{cc}
D_{1} f_{1} F_{1} & D_{2} f_{2} F_{2} \\
f_{1} F_{1} & f_{2} F_{2}
\end{array}\right| & \rightarrow\left|\begin{array}{cc}
\left(D_{1}+\alpha\right) f_{1} F_{1} & \left(D_{2}+\beta\right) f_{2} F_{2} \\
f_{1} F_{1} & f_{2} F_{2}
\end{array}\right| \\
& =\left|\begin{array}{cc}
D_{1} f_{1} F_{1} & \left(D_{2}+(\beta-\alpha)\right) f_{2} F_{2} \\
f_{1} F_{1} & f_{2} F_{2}
\end{array}\right| .
\end{aligned}
$$

It is evident that the determinant is only invariant under the transformation if $\alpha=\beta$.

\section{MODULAR COVARIANCE}

We are now in a position to study the modular covariance properties of the PWJ wave functions. For this purpose, and to simplify the discussion somewhat, we assume that we are considering one of the CF ground states at filling fraction $v=$ $\frac{n}{2 p n+1}$. That is, we assume that we have a state with $n$ filled $\Lambda$ 
levels, and everything above unoccupied. In this work we will devote most attention to the $\mathcal{S}$ transform $\tau \rightarrow-\frac{1}{\tau}$ since that is the more complicated of the two. At the end of this section, the $\mathcal{T}$ transform $\tau \rightarrow \tau+1$ will be briefly discussed.

Before we deal with the many-body state, let us review how single particle orbitals transform under the $\mathcal{S}$ transform. An $S$ transform sends $\tau \rightarrow-\frac{1}{\tau}$ and affects the LLL single particle orbitals from (5) as

$$
\begin{aligned}
\phi_{i, 0}^{(M)}\left(x, y,-\frac{1}{\tau}\right)= & e^{-l 2 \pi M y x} \sqrt{\frac{\tau}{|\tau|}} \frac{1}{\sqrt{M}} \\
& \times \sum_{k} e^{\imath 2 \pi \frac{k i}{M}} \phi_{k, 0}^{(M)}(-y, x, \tau) .
\end{aligned}
$$

The higher order higher LL orbitals in (6) similarly transform as

$$
\begin{aligned}
\phi_{i, n}^{(M)}\left(x, y,-\frac{1}{\tau}\right)= & e^{-l 2 \pi M y x}\left(\frac{\tau}{|\tau|}\right)^{n+\frac{1}{2}} \frac{1}{\sqrt{M}} \\
& \times \sum_{k} e^{t 2 \pi \frac{k i}{M}} \phi_{k, n}^{(M)}(-y, x, \tau) .
\end{aligned}
$$

In the above equations we note that $\tau \rightarrow-\frac{1}{\tau}$ effectively sends $y \rightarrow x \rightarrow-y$ and maps $\phi_{i, n}^{(M)}$ into a Fourier sum $\sum_{k} e^{i 2 \pi \frac{k i}{M}} \phi_{k, n}^{(M)}$. We may also identify the factor $e^{-l 2 \pi M y x}$ as the gauge transformation related with the coordinate change and $\left(\frac{\tau}{|\tau|}\right)^{n+\frac{1}{2}}$ can be interpreted as the conformal weight of the orbital. The extra factors of $\frac{\tau}{|\tau|}$ for $n>0$ can be understood by noting that the derivative operator $\partial_{z}^{(x, y, \tau)}$ transforms as

$$
\begin{aligned}
\partial_{z}^{\left(x, y,-\frac{1}{\tau}\right)} & =\frac{|\tau|}{\bar{\tau} L 2 \imath \tau_{2}}\left(\bar{\tau} \partial_{y}+\partial_{x}\right) \\
& =\left(\frac{\tau}{|\tau|}\right) \partial_{z}^{(-y, x, \tau)} .
\end{aligned}
$$

In this calculation we used that $\tau_{2} \rightarrow \frac{\tau_{2}}{|\tau|^{2}}, L=\sqrt{\frac{2 \pi N_{\phi}}{\tau_{2}}} \rightarrow$ $|\tau| \sqrt{\frac{2 \pi N_{\phi}}{\tau_{2}}}=|\tau| L$ and that $\bar{\tau} \rightarrow-\frac{1}{\bar{\tau}}=\frac{-\tau}{|\tau|^{2}}$. Thus, the ladder operator $a_{\tau}^{\dagger}(x, y, \tau)=-\sqrt{2}\left(\partial_{z}-\frac{\bar{\tau}}{2} L y\right)$ transforms as

$$
\begin{aligned}
a_{\tau}^{\dagger}\left(x, y,-\frac{1}{\tau}\right) & =-\sqrt{2}\left(\frac{\tau}{|\tau|}\right)\left(\partial_{z}^{(-y, x, \tau)}+\frac{1}{2} L y\right) \\
& =e^{-l 2 \pi M y x}\left(\frac{\tau}{|\tau|}\right) a_{\tau}^{\dagger}(-y, x, \tau) e^{\imath 2 \pi M y x} .
\end{aligned}
$$

By applying (26) and (24) to $\phi_{j, n}=a_{\tau}^{\dagger n} \phi_{j, 0}$ then (25) is directly obtained. From this also follows that $J_{j}^{p}$, which is used in the CF construction, is invariant under $\tau \rightarrow-\frac{1}{\tau}$ since they contain products of $\sigma_{\frac{1}{2}, \frac{1}{2}}^{(1)}\left(z_{i j}\right)$ which are an $M=1$ representation.

\section{A. A modular invariant $\mathbf{C F}$ wave function}

To make the discussion in the following subsection a little bit cleaner, we spend some time in this section defining a CF wave function that is invariant under $\mathcal{S}$ transforms in its unprojected form. We do this, since if we can find one wave function $\psi$ that is invariant under $S$, we can then build the family of $q$-fold degenerate wave functions from this template, as eigenstates of either $T_{1}=T\left(\frac{L}{N_{\phi}}\right)$ or $T_{2}=T\left(\frac{\tau L}{N_{\phi}}\right)$.

In practice, if we assume that $\psi$ is a wave function that is modular invariant, we may define the states $\psi_{j}=T_{2}^{j} P_{1,0} \psi$ and $\varphi_{l}=T_{1}^{-l} P_{2,0} \psi$. Here

$$
P_{m, l}=\frac{1}{q} \sum_{j} e^{i 2 \pi \frac{-l j}{q}} T_{m}^{j},
$$

is a projector onto the basis defined by $T_{m}$ and it satisfies $\sum_{l=1}^{q} P_{m, l}=1 \quad$ and $\quad P_{m, l} P_{m, k}=P_{m, l} \delta_{l, k}$. Since $T_{1} T_{2}=$ $T_{2} T_{1} e^{\imath 2 \pi \frac{p}{q}}$ we have that

$$
\begin{aligned}
& \psi_{l} \propto \frac{1}{\sqrt{q}} \sum_{j} e^{i 2 \pi \frac{-p l j}{q}} \varphi_{j} \\
& \varphi_{j} \propto \frac{1}{\sqrt{q}} \sum_{j} e^{i 2 \pi \frac{p l j}{q}} \psi_{j},
\end{aligned}
$$

where the $\propto$ is inserted since $\psi_{l}$ and $\varphi_{j}$ might not be properly normalized with respect to each other. Now, by applying the $S$ transform, which transforms $T_{1} \rightarrow T_{2} \rightarrow T_{2}^{-1}$, we find that

$$
\begin{aligned}
\psi_{l} & =T_{2}^{l} P_{1,0} \psi \\
& \rightarrow T_{1}^{-l} P_{2,0} \psi \\
& =\varphi_{l} \propto \frac{1}{\sqrt{q}} \sum_{j} e^{l 2 \pi \frac{p l j}{q}} \psi_{j}
\end{aligned}
$$

which shows that the set of $q$ wave functions $\psi_{l}$ is closed under $\mathcal{S}$. It thus remains to be seen that $\psi$ itself is invariant under $\mathcal{S}$, and this will be the focus of the following sections.

\section{B. Unprojected CF}

According to the argument of the previous section, it is sufficient to show modular covariance if we can find one CF wave function that is invariant under the $\mathcal{S}$ transform. For this purpose we note that if we choose $\psi_{v=1}^{2 p}$ instead of $\psi_{v=\frac{1}{2 p}}$ in (18) then the center of mass part and the Jastrow factors $\sigma_{\frac{1}{2}, \frac{1}{2}}\left(z_{i j}\right)$ are all manifestly invariant under these transformations, up to constant factors and phases. The determinant $\chi_{n}$ can be made invariant in two different but equivalent ways. The first is to argue that if one fills a $\Lambda$ level completely, it will also be filled after the $S$ transform, thus ensuring the invariance. The second, which will make the later discussion of the PWJ projection much cleaner, is to build $\chi_{n}$ from orbitals that themselves are invariant under the $\mathcal{S}$ transform.

By choosing the $\chi_{n}$ orbitals as linear combinations lattice coherent states (LCS)

$$
\rho_{n, m}(z)=\sigma_{\frac{1}{2}, \frac{1}{2}}\left(z-\frac{1}{M}(n+\tau m)\right)^{M},
$$

one can ensure that each orbital is invariant under $\mathcal{S}$. These states where introduced by Haldane in Ref. [3] as a possible way to construct maximally localized wave functions and were later studied in detail in Ref. [33]. They have the property that they have all their zeros at the same position $z=L(n+\tau m)$ and transform as $\rho_{n, m} \rightarrow \rho_{m,-n}$ under modular $\mathcal{S}$ transformations. By choosing our orbitals from linear 
combinations of LCS as

$$
\tilde{\rho}_{n, m}=\rho_{n, m}+\rho_{m,-n}+\rho_{-n,-m}+\rho_{-m, n},
$$

we ensure that all the orbitals transform trivially under the $\mathcal{S}$ transform. These are examples of eigenstates for certain finite rotations. The LCS forms an over complete $M \times M$ lattice of states and there are thus roughly $M^{2} / 4$ acceptable choices for $\tilde{\rho}_{n, m}$. Since $M^{2} / 4>M$ these states are enough to fill the lowest $\Lambda$ level and thus all also of the higher $\Lambda$ levels by the action of raising operators.

\section{Exactly projected $\mathbf{C F}$}

To prove that the exactly projected states have good modular properties, it is sufficient to show that the modular transformation commutes with the projector $P_{\mathrm{LLL}}$. This is straightforward due to equations (24) and (25). These equations namely show that the modular transformation never mixes states between Landau levels and thus trivially commutes with the Landau level projection.

A more formal proof of the same is to note that $P_{\mathrm{LLL}}$ can formally be written as $P_{\mathrm{LLL}}=\prod_{n=1}^{\infty}\left(1-\frac{a^{\dagger} a}{n}\right)$ where $a^{\dagger}$ and $a$ are the operators in (2). Using the result from (26) we see that $a_{\tau}^{\dagger} a_{\tau} \rightarrow e^{-t 2 \pi M y x} a_{\tau}^{\dagger} a_{\tau} e^{22 \pi M y x}$ only contains the overall gauge transformation $e^{\imath 2 \pi M y x}$, and so $P_{\mathrm{LLL}} \rightarrow e^{-t 2 \pi M y x} P_{\mathrm{LLL}} e^{2 \pi M y x}$ under the $\mathcal{S}$ transform. This shows that $P_{\text {LLL }}$ commutes with $\mathcal{S}$ up to the ever present gauge transformation.

\section{PWJ projected CF}

We now turn our attention to the PWJ projected CF state, where we are especially interested in the transformation properties of $\tilde{f}_{n}$ and $F_{j}^{p}$ as defined in (20) and (21). We here assume, following the discussion in Secs. VI A and VIB that $f_{0}$ is chosen from the set of lattice coherent states (27). This is not strictly necessary for the arguments below, however, since the LCS in (28) are themselves invariant under $\mathcal{S}$ transforms, it saves us from keeping track of additional Fourier sums that would be present if, e.g., the orbitals in (24) where used.

For $f_{j} \equiv f_{0}\left(z_{j}\right)$ and $F_{j}$ we have the respective transformations, again with constant faces removed

$$
f_{j}^{(M)} \rightarrow e^{\imath \tau \tau M z_{j}^{2}} f_{j}^{(M)},
$$

and

$$
\begin{aligned}
F_{j} \rightarrow e^{\imath \tau \pi \sum_{l}\left(z_{j}-z_{l}\right)^{2}} F_{j} & =e^{l \tau \pi\left(N_{e} z_{j}^{2}-2 z_{j} Z+\sum_{l} z_{l}^{2}\right)} F_{j} \\
& =e^{\imath \tau \pi\left(\left(N_{e}-1\right) z_{j}^{2}-2 z_{j} Z_{j}+\sum_{l \neq j} z_{l}^{2}\right.} F_{j} .
\end{aligned}
$$

The combined transformation is thus

$$
\begin{aligned}
\tilde{D}_{j}^{n} f_{j} F_{j}^{p} \rightarrow & \tilde{D}_{j}^{n}\left(e^{\imath \pi \tau M z_{j}^{2}} f_{j}\right) \\
& \times\left(e^{l \tau \pi p\left(\left(N_{e}-1\right) z_{j}^{2}-2 z_{j} Z_{j}+\sum_{l \neq j} z_{l}^{2}\right)} F_{j}^{p}\right) .
\end{aligned}
$$

The factor of $e^{\imath \tau \tau z_{j}^{2}}$ can be traced to the $\vartheta$-function relation

$$
\vartheta_{a, b}\left(z \mid-\frac{1}{\tau}\right)=\sqrt{-\imath \tau} e^{\imath \pi \tau z^{2}} e^{\imath 2 \pi b a} \vartheta_{b,-a}(\tau z \mid \tau)
$$

after applying the scaling $z \rightarrow M z$ and $\tau \rightarrow \frac{\tau}{M}$.
Let us first consider the simplest case of $n=1$ where we define $\gamma=e^{\imath \pi \tau M z_{j}^{2}} e^{l \tau \pi p\left(\left(N_{e}-1\right) z_{j}^{2}-2 z_{j} Z_{j}+\sum_{l \neq j} z_{l}^{2}\right)}$. This yields

$$
\begin{aligned}
\tilde{D}_{j} f_{j} F_{j}^{p} \rightarrow & \gamma^{-1}\left(-2 p N_{e} \tilde{\partial}_{z_{j}}+2 M \hat{\partial}_{z_{j}}\right)\left(e^{\imath \pi \tau M z_{j}^{2}} f_{j}\right) \\
& \times\left(e^{\imath \tau \pi p\left(\left(N_{e}-1\right) z_{j}^{2}-2 z_{j} Z_{j}+\sum_{l \neq j} z_{l}^{2}\right)} F_{j}^{p}\right) \\
= & -2 p N_{e}\left(\tilde{\partial}_{z_{j}}+\imath 2 \pi \tau M z_{j}\right) f_{j} F_{j}^{p} \\
& +2 M\left(\hat{\partial}_{z_{j}}+\imath \tau \pi 2 p\left(\left(N_{e}-1\right) z_{j}-Z_{j}\right)\right) f_{j} F_{j}^{p} \\
= & \tilde{D}_{j} f_{j} F_{j}^{p}-\imath 4 \pi \tau M p Z f_{j} F_{j}^{p},
\end{aligned}
$$

where we see an extra term $-\imath \tau \pi 4 M p Z f_{j} F_{j}^{p}$ appearing. This term can then be removed under row addiction of the determinant. This is since it is proportional to $Z f_{j} F_{j}^{p}$ and $Z$ is independent of the $j$ index.

For general $n$ we cannot use the trick employed above since $\left[\partial_{z},\left[\partial_{z}, z^{2}\right]\right]=2 \neq 0$, which means that the factors $\tilde{\partial}_{z_{j}} \rightarrow$ $\tilde{\partial}_{z_{j}}+\imath 2 \pi \tau M z_{j}$ and $\hat{\partial}_{z_{j}} \rightarrow \hat{\partial}_{z_{j}}+\imath \tau \pi 2 p\left(\left(N_{e}-1\right) z_{j}-Z_{j}\right)$ can only in the $n=1$ case be directly combined to $\tilde{D} \rightarrow \tilde{D}-$ $\imath \tau \pi 4 M p Z$. For the $n=2$ case, one may after some algebra conclude that

$$
\begin{aligned}
\tilde{D}^{2} \rightarrow & \tilde{D}^{2}+\imath \pi \tau 8 M p Z \tilde{D}-16(\pi \tau M p)^{2} Z^{2} \\
& -8 \imath \pi \tau M p\left(M\left(N_{e}-1\right)+N_{e}^{2} p\right) .
\end{aligned}
$$

Here we see that we still only get terms that depend on $Z$ and $\tilde{D}$, and they can all be removed by row addition. By Mathematica calculations we can confirm up to $n=10$, and we believe it holds in general that the general transformation that takes place is

$$
\tilde{D}^{n} \rightarrow \sum_{k=0}^{n} \sum_{l=0}^{\left\lfloor\frac{k}{2}\right\rfloor} A_{k, l} Z^{k-2 l} \tilde{D}^{n-k} \alpha^{k} \beta^{l} .
$$

The constants in the expression are $\alpha=\imath \pi \tau 4 M p, \beta=$ $\frac{M\left(N_{e}-1\right)+N_{e}^{2} p}{l \pi \tau 2 M p}$ and $A_{k, l}$ is defined as

$$
\begin{aligned}
A_{k, 0} & =\left(\begin{array}{l}
n \\
k
\end{array}\right) \\
A_{k, l} & =-\frac{A_{2 l, l-1}}{l}\left(\begin{array}{l}
n-2 l \\
k-2 l
\end{array}\right) .
\end{aligned}
$$

Again, since the extra terms that are generated are all proportional to powers of $Z$ and $\tilde{D}$, they can all be removed by row addition in the determinant is the CF state is proper. This proves that the PWJ wave functions transform nicely under modular $\mathcal{S}$ transformations.

\section{E. Modular $\mathcal{T}$ transforms}

For completeness we here discuss the effect of the $\mathcal{T}$ transform $\tau \rightarrow \tau+1$ on the PWJ states. Since the $\mathcal{S}$ and $\mathcal{T}$ transformations in general do not commute, it means that the LCS orbitals that were used in the analysis of the $\mathcal{S}$ transform are not appropriate here. Fortunately, the orbitals in (5) are already invariant under $\mathcal{T}$ since they transform as

$$
\phi_{i}^{(M)}(x, y, \tau+1)=e^{\imath \pi M y^{2}} e^{\imath \pi \frac{i^{2}}{M}} e^{\imath \pi i M} \phi_{i}^{(M)}\left(x+y+\frac{M}{2}, y, \tau\right) .
$$


Here the factor $e^{\imath \pi M y^{2}}$ is related to the gauge transformation associated with the coordinate change $x \rightarrow x+y$, and the extra shift of $\frac{M}{2}$ is related to the difference in area $\pi M \ell_{B}^{2}$ covered by the winding paths $t(\tau L)$ and $t(L(1+\tau))$.

By virtue of (29) is follows that any (unprojected) CF wave function constructed in terms these orbitals is automatically invariant under the $\mathcal{T}$ transform. Just as in Sec. VIC it also follows that exactly projected $\mathrm{CF}$ wave functions are invariant under the $\mathcal{T}$ transform, since the transformation commutes with the LLL projector.

Also for the PWJ projected wave functions, the proof is straightforward, since the gauge factor $e^{l \pi M y^{2}}$ arises exclusively from the Gaussian factor $e^{l \pi \tau M y^{2}}$. Since this factor is not present in the holomorphic function $f(z)$, it is therefore not touched by the $\tilde{D}$ operator [which transforms as $\tilde{D}^{(x, y, \tau+1)}=$ $\tilde{D}^{(x+y, y, \tau)}$ in accordance with (29)], and thus the PWJ projected states are also invariant under the $\mathcal{T}$ transform.

\section{DISCUSSION}

In this paper we have shown explicitly that the $\mathrm{CF}$ wave functions have proper modular properties on the torus. As part of this work we have also reformulated the PWJ method in $\tau$ gauge, which is the natural gauge choice for the torus. We have along the way exposed a series of analytical expressions for the projected states that we hope will be useful for future studies of composite fermions on the torus. One limitation of the original PWJ formulation is that it is not applicable for reverse flux states, and we especially hope that this is a step in the direction of extending the PWJ method to this class of $\mathrm{CF}$ wave functions.

\section{ACKNOWLEDGMENTS}

I would like to thank J. K. Jain, S. Pu, and H. Hansson for enlightening discussions and for the encouragement to write this paper. I gratefully acknowledge financial support from Science Foundation Ireland Principal Investigator Award 12/IA/1697. This work is part of the D-ITP consortium, a program of the Netherlands Organisation for Scientific Research (NWO) that is funded by the Dutch Ministry of Education, Culture and Science (OCW).

\section{APPENDIX A: THE LLL PROJECTION OF $\tilde{y}$}

We know from the work of Girvin and Jach [11] that we may write the LLL projection as

$$
P_{\mathrm{LLL}} \bar{z} G_{s} f_{s}(z)=G_{s}\left(2 \partial_{z}\right) f_{s}(z),
$$

where $G_{s}=e^{-\frac{z \bar{z}}{4}}$ and $f_{s}(z)$ is a polynomial in $z$. Any wave function in symmetric gauge can be transformed into $\tau$ gauge with the action of the unitary operator $U_{s \rightarrow \tau}=e^{-\imath \pi N_{\phi} x y}$ in such a way that

$$
U_{s \rightarrow \tau} G_{s} f_{s}(z)=G_{\tau} f_{\tau}(z),
$$

where $G_{\tau}=e^{\imath \pi \tau N_{\phi} y^{2}}$ and $f_{\tau}(z)=e^{-\frac{z^{2}}{4}} f_{s}(z)$ is also a holomorphic polynomial. Technically, also $P_{\text {LLL }}$ is gauge dependent but we suppress that in the analysis below. Applying $U_{s \rightarrow \tau}$ to the left and right hand sides of Eq. (A1) now gives

$$
P_{\mathrm{LLL}} \bar{z} G_{\tau} f_{\tau}(z)=G_{\tau} e^{-\frac{z^{2}}{4}}\left(2 \partial_{z}\right) e^{\frac{z^{2}}{4}} f_{\tau}(z)
$$

which after pulling the $\partial_{z}$ through the $e^{\frac{z^{2}}{4}}$ gives $P_{\mathrm{LLL}} \bar{z} G_{\tau} f_{\tau}(z)=G_{\tau}\left(2 \partial_{z}+z\right) f_{\tau}(z)$. Finally after moving $G_{\tau} z f_{\tau}(z)$ to the left-hand side and using that $P_{\mathrm{LLL}} G_{\tau} z f_{\tau}(z)=$ $G_{\tau} z f_{\tau}(z)$ while identifying $\bar{z}-z=-2 \imath \tilde{y}$, we have

$$
P_{\mathrm{LLL}} \tilde{y} G_{\tau} f_{\tau}(z)=G_{\tau}\left(l \partial_{z}\right) f_{\tau}(z)
$$

just as in the main text. The generalization to higher powers of $\tilde{y}$ is straightforward since we can write

$$
\begin{aligned}
P_{\mathrm{LLL}} \tilde{y}^{n} G_{\tau} f_{\tau}(z)= & \frac{1}{(-2 \imath)^{n}} P_{\mathrm{LLL}}(\bar{z}-z)^{n} G_{\tau} f_{\tau}(z) \\
= & \frac{1}{(-2 \imath)^{n}} P_{\mathrm{LLL}} \sum_{k=0}^{n}\left(\begin{array}{l}
n \\
k
\end{array}\right) \bar{z}^{k}(-z)^{n-k} G_{\tau} f_{\tau}(z) \\
= & \frac{1}{(-2 \imath)^{n}} G_{\tau} \sum_{k=0}^{n}\left(\begin{array}{l}
n \\
k
\end{array}\right) e^{-\frac{z^{2}}{4}}\left(2 \partial_{z}\right)^{k} \\
& \times e^{\frac{z^{2}}{4}}(-z)^{n-k} f_{\tau}(z) \\
= & \frac{1}{(-2 \imath)^{n}} G_{\tau} \sum_{k=0}^{n}\left(\begin{array}{l}
n \\
k
\end{array}\right)\left(2 \partial_{z}+z\right)^{k}(-z)^{n-k} f_{\tau}(z) .
\end{aligned}
$$

For the second row we expanded $(\bar{z}-z)^{n}$, for the third we used the rule $\bar{z}^{k} \rightarrow\left(2 \partial_{z}\right)^{k}$, and for the last row that $e^{-\frac{z^{2}}{4}}\left(2 \partial_{z}\right)^{k} e^{\frac{z^{2}}{4}}=\left(2 \partial_{z}+z\right)^{k}$. We will now prove that Eq. (A4) can be rewritten as the more elegant

$$
P_{\mathrm{LLL}} \tilde{y}^{n} G_{\tau} f_{\tau}(z)=\frac{1}{(-2 \iota)^{n}} G_{\tau} H_{n}\left(\partial_{z}\right) f_{\tau}(z),
$$

where $H_{n}(x)$ is a Hermite polynomial. The proof uses that the Hermite polynomial satisfies the relation $H_{n+1}(x)=$ $2 x H_{n}(x)-H_{n}^{\prime}(x)$. Since $H_{n}$ has an operator $\partial_{z}$ as argument, we can implement the derivative with respect to $\partial_{z}$ as $\frac{\partial}{\partial_{\partial_{2}}} H_{n}\left(\partial_{z}\right)=$ $\left[H_{n}\left(\partial_{z}\right), z\right]$. We then get the equation

$$
H_{n+1}\left(\partial_{z}\right)=2 \partial_{z} H_{n}\left(\partial_{z}\right)-\left[H_{n}\left(\partial_{z}\right), z\right]
$$

where we propose that

$$
H_{n}\left(\partial_{z}\right) \stackrel{?}{=} \sum_{k=0}^{n}\left(\begin{array}{l}
n \\
k
\end{array}\right)\left(2 \partial_{z}+z\right)^{k}(-z)^{n-k}
$$

is a solution. We construct a proof by induction. First we show that $H_{1}\left(\partial_{z}\right)=(-z)+\left(2 \partial_{z}+z\right)=2 \partial_{z}$ is trivially true. After some algebra we can show that (A4) satisfies the recursion 
relation (A5). This is since

$$
\begin{aligned}
2 \partial_{z} H_{n}\left(\partial_{z}\right)-\left[H_{n}\left(\partial_{z}\right), z\right] & =\sum_{k=0}^{n}\left(\begin{array}{l}
n \\
k
\end{array}\right)\left[2 \partial_{z}\left(2 \partial_{z}+z\right)^{k}(-z)^{n-k}+\left(2 \partial_{z}+z\right)^{k}(-z)^{n+1-k}+z\left(2 \partial_{z}+z\right)^{k}(-z)^{n-k}\right] \\
& =\sum_{k=0}^{n}\left(\begin{array}{l}
n \\
k
\end{array}\right)\left(2 \partial_{z}+z\right)^{k+1}(-z)^{n-k}+\sum_{k=0}^{n}\left(\begin{array}{l}
n \\
k
\end{array}\right)\left(2 \partial_{z}+z\right)^{k}(-z)^{n+1-k} \\
& =\left\{\sum_{k=1}^{n+1}\left(\begin{array}{c}
n \\
k-1
\end{array}\right)+\sum_{k=0}^{n}\left(\begin{array}{l}
n \\
k
\end{array}\right)\right\}\left(2 \partial_{z}+z\right)^{k}(-z)^{n+1-k} \\
& =\sum_{k=0}^{n+1}\left(\begin{array}{c}
n+1 \\
k
\end{array}\right)\left(2 \partial_{z}+z\right)^{k}(-z)^{n+1-k}=H_{n+1}\left(\partial_{z}\right),
\end{aligned}
$$

where, on line four, we used that $\left(\begin{array}{c}n \\ n+1\end{array}\right)=\left(\begin{array}{c}n \\ -1\end{array}\right)=0$. This concludes the proof.

\section{APPENDIX B: THE PROJECTION OPERATOR}

In this section we investigate the effect of the GJ trick on the $n$th LL wave function $\phi_{j, n}^{(M)}$ as defined in (6), where it is also understood that this is always multiplied with a $N_{\phi}-M$ flux wave function. If we strip off the leading Gaussian we have the wave function

$$
\begin{aligned}
f_{j, n}^{(M)}= & \mathcal{N}_{n} \sum_{k \in \mathbb{Z}+\frac{j}{N_{\phi}}} e^{\imath \pi M \tau k^{2}} \\
& \times e^{i 2 \pi M k \frac{z}{L}} H_{n}\left(\tilde{y}+\tau_{2} L k\right) .
\end{aligned}
$$

As mentioned in the previous section, we cannot simply replace $\tilde{y} \rightarrow \iota \partial_{z}$, but the rule is rather that $\tilde{y}^{n} \rightarrow \frac{1}{(-2 \iota)^{n}} H_{n}\left(\partial_{z}\right)$. By expanding the Hermite polynomial in powers of $\tilde{y}+\tau_{2} L k$ we have

$$
\begin{aligned}
H_{n}\left(\tilde{y}+\tau_{2} L k\right) & =\sum_{m=0}^{\left\lfloor\frac{n}{2}\right\rfloor} g_{n, m}\left(\tilde{y}+\tau_{2} L k\right)^{n-2 m} \\
& =\sum_{m=0}^{\left\lfloor\frac{n}{2}\right\rfloor} g_{n, m} \sum_{r=0}^{n-2 m}\left(\begin{array}{c}
n-2 m \\
r
\end{array}\right) \tilde{y}^{n-2 m-r}\left(\tau_{2} L k\right)^{r},
\end{aligned}
$$

where we used the expansion $H_{n}(x)=\sum_{m=0}^{\left\lfloor\frac{n}{2}\right\rfloor} g_{n, m} x^{n-2 m}$ and $g_{n, m}=\frac{n !(-1)^{m} 2^{n-2 m}}{m !(n-2 m) !}$. We note that we can write $\left(\tau_{2} L k\right)^{r} e^{\imath 2 \pi M k_{L}} \cdot 1=\left(-\imath \partial_{z} \frac{N_{\phi}}{M}\right)^{r} e^{r 2 \pi M k_{L}} \cdot 1 . \quad$ This allows us to write (B1) as

$\hat{f}_{j, n}^{(M)}=\frac{\mathcal{N}_{n}}{\mathcal{N}_{0}} \sum_{m=0}^{\left\lfloor\frac{n}{2}\right\rfloor} g_{n, m} \sum_{r=0}^{n-2 m}\left(\begin{array}{c}n-2 m \\ r\end{array}\right) \tilde{y}^{n-2 m-r}\left[\left(-l \partial_{z} \frac{N_{\phi}}{M}\right)^{r} f_{j, 0}\right]$,

where the derivatives within the square bracket box only act on $f_{j, 0}$. After the projection step this becomes

$$
\begin{aligned}
\hat{f}_{j, n}^{(M)}= & \frac{\mathcal{N}_{n}}{\mathcal{N}_{0}} \sum_{m=0}^{\left\lfloor\frac{n}{2}\right\rfloor} g_{n, m} \sum_{r=0}^{n-2 m}\left(\begin{array}{c}
n-2 m \\
r
\end{array}\right) \\
& \times \frac{H_{n-2 m-r}\left(\partial_{z}\right)}{(-2 \iota)^{n-2 m-r}}\left[\left(-\partial_{z} \frac{N_{\phi}}{M}\right)^{r} f_{j, 0}\right] .
\end{aligned}
$$

However, due to a clever resummation of Hermite polynomials, which we will not demonstrate, we have the much cleaner result

$$
\hat{f}_{j, n}^{(M)}=\sum_{k=0}^{n}\left(\begin{array}{l}
n \\
k
\end{array}\right) M^{n-k} \partial_{z}^{n-k}\left[\left(-N_{\phi}\right)^{k} \partial_{z}^{k} f_{j, 0}\right],
$$

where we have once again dropped $\frac{(22)^{n}}{M^{n}} \frac{\mathcal{N}_{n}}{\mathcal{N}_{0}}$ just as in the main text.

\section{APPENDIX C: OPERATORS WITH PERIODIC BOUNDARY CONDITIONS}

Similarly to the relation $e^{l \pi \tau M y^{2}} f_{j, n}^{(M)}=\phi_{j, n}^{(M)}$ in the main text, we may now define an operator equivalent of the LLL projector $e^{l \pi \tau N_{\phi} y^{2}} \hat{f}_{j, n}^{(M)}=\hat{\phi}_{j, n}^{(M)} e^{l \pi \tau\left(N_{\phi}-M\right) y^{2}}$ for a general $n$th Landau level. We may express $\hat{\phi}_{j, n}^{(M)}$ as a series expansion in

$$
\hat{g}_{n}=\left.\hat{f}_{n}^{(M)}\right|_{f_{0} \rightarrow \phi_{0}}=\left(M \partial_{z}-\tilde{\partial}_{z} N_{\phi}\right)^{n} \phi_{0},
$$

where we simply replace the $f_{0}$ in $\hat{f}_{j, n}^{(M)}$ by $\phi_{0}$. It is straightforward to show that the operator $\hat{g}_{n}$ satisfies the desired periodicity boundary conditions

$$
e^{i 2 \pi N_{\phi} x} \tilde{t}(\tau L) \hat{g}_{n}=\hat{g}_{n} \tilde{t}(\tau L) e^{\imath 2 \pi\left(N_{\phi}-M\right) x}
$$

by repeating the arguments that were used in conjunction with Eq. (16). The only difference is that now it is an exponential of $x$ and not $z$ that is considered. However, since $\left[\partial_{z},\left[\partial_{z}, x\right]\right]=$ $0=\left[\partial_{z},\left[\partial_{z}, z\right]\right]$ the calculation is identical.

Considering now the function

$$
\hat{\phi}_{n}^{(M)}=e^{\imath \pi \tau N_{\phi} y^{2}} \hat{f}_{n}^{(M)} e^{-l \pi \tau\left(N_{\phi}-M\right) y^{2}},
$$

we can use (C1) to argue that $\hat{\phi}_{n}^{(M)} \neq \hat{g}_{n}$ but that there will also be subleading terms proportional to $\hat{g}_{n-2}, \hat{g}_{n-4}, \ldots, \hat{g}_{0}$. Unlike the arguments that were used in conjunction with Eq. (16) we are now pulling exponentials of $y^{2}$ through $\hat{D}$, and since $\left[\partial_{z},\left[\partial_{z}, y^{2}\right]\right] \neq 0$ the shifts of $\partial_{z}$ and $\tilde{\partial}_{z}$ cannot be applied independently. This is what leads to the subleading terms. If we define $\chi=M\left(N_{\phi}-M\right) N_{\phi} \frac{\pi \tau}{2 l \tau_{2}^{2} L^{2}}=\frac{M\left(N_{\phi}-M\right) \tau}{4 l \tau_{2}}$ then we may explicitly show that

$$
\begin{aligned}
& \hat{\phi}_{1}=\hat{g}_{1} \\
& \hat{\phi}_{2}=\hat{g}_{2}+1 \chi \hat{g}_{0}
\end{aligned}
$$




$$
\begin{aligned}
& \hat{\phi}_{3}=\hat{g}_{3}+3 \chi \hat{g}_{1} \\
& \hat{\phi}_{4}=\hat{g}_{4}+6 \chi \hat{g}_{2}+3 \chi^{2} \hat{g}_{0} \\
& \hat{\phi}_{5}=\hat{g}_{5}+10 \chi \hat{g}_{3}+15 \chi \hat{g}_{1} \\
& \hat{\phi}_{6}=\hat{g}_{6}+15 \chi \hat{g}_{4}+45 \chi^{2} \hat{g}_{2}+15 \chi^{3} \hat{g}_{0} \\
& \hat{\phi}_{7}=\hat{g}_{7}+21 \chi \hat{g}_{5}+105 \chi^{2} \hat{g}_{3}+105 \chi^{3} \hat{g}_{1} \\
& \hat{\phi}_{8}=\hat{g}_{8}+28 \chi \hat{g}_{6}+210 \chi^{2} \hat{g}_{4}+420 \chi^{3} \hat{g}_{2}+105 \chi^{4} \hat{g}_{0} .
\end{aligned}
$$

This may be summarized as

$$
\hat{\phi}_{n}=\sum_{k=0}^{\left\lceil\frac{n}{2}\right\rceil} T(n, k) \chi^{k} \hat{g}_{n-2 k},
$$

where $T(n, k)$ is the triangle of Bessel numbers (OEIS series A100861) [31].
[1] R. B. Laughlin, Phys. Rev. Lett. 50, 1395 (1983).

[2] F. D. M. Haldane, Phys. Rev. Lett. 51, 605 (1983).

[3] F. D. M. Haldane and E. H. Rezayi, Phys. Rev. B 31, 2529 (1985).

[4] G. Moore and N. Read, Nucl. Phys. B 360, 362 (1991).

[5] R. H. Morf, Phys. Rev. Lett. 80, 1505 (1998).

[6] E. H. Rezayi and F. D. M. Haldane, Phys. Rev. Lett. 84, 4685 (2000).

[7] S. Pu, Y.-H. Wu, and J. K. Jain, Phys. Rev. B 96, 195302 (2017).

[8] J. K. Jain, Phys. Rev. Lett. 63, 199 (1989).

[9] G. Dev and J. K. Jain, Phys. Rev. Lett. 69, 2843 (1992).

[10] M. Hermanns, Phys. Rev. B 87, 235128 (2013).

[11] S. M. Girvin and T. Jach, Phys. Rev. B 29, 5617 (1984).

[12] J. K. Jain and R. K. Kamilla, Int. J. Mod. Phys. B 11, 2621 (1997).

[13] M. Hermanns, J. Suorsa, E. J. Bergholtz, T. H. Hansson, and A. Karlhede, Phys. Rev. B 77, 125321 (2008).

[14] M. Fremling, T. H. Hansson, and J. Suorsa, Phys. Rev. B 89, 125303 (2014).

[15] T. H. Hansson, M. Hermanns, S. H. Simon, and S. F. Viefers, Rev. Mod. Phys. 89, 025005 (2017).

[16] M. P. Zaletel and R. S. K. Mong, Phys. Rev. B 86, 245305 (2012).

[17] J. Kjäll, E. Ardonne, V. Dwivedi, M. Hermanns, and T. H. Hansson, J. Stat. Mech.: Theor. Exp. (2018) 053101.
[18] M. P. Zaletel, R. S. K. Mong, F. Pollmann, and E. H. Rezayi, Phys. Rev. B 91, 045115 (2015).

[19] M. Greiter, V. Schnells, and R. Thomale, Phys. Rev. B 93, 245156 (2016).

[20] S. Pu, M. Fremling, and J. K. Jain, Phys. Rev. B 98, 075304 (2018).

[21] J. Wang, S. D. Geraedts, E. H. Rezayi, and F. D. M. Haldane, arXiv: 1710.09729.

[22] M. Fremling, N. Moran, J. K. Slingerland, and S. H. Simon, Phys. Rev. B 97, 035149 (2018).

[23] S. D. Geraedts, J. Wang, E. H. Rezayi, and F. D. M. Haldane, Phys. Rev. Lett. 121, 147202 (2018).

[24] N. Read, Phys. Rev. B 79, 045308 (2009).

[25] N. Read, arXiv:0807.3107.

[26] N. Read and E. H. Rezayi, Phys. Rev. B 84, 085316 (2011).

[27] F. D. M. Haldane, J. Math. Phys. 59, 071901 (2018).

[28] F. D. M. Haldane, J. Math. Phys. 59, 081901 (2018).

[29] M. Fremling, J. Phys. A 50, 015201 (2016).

[30] This suppressed factor is irrelevant when one only considers product states of CFs (or more generally when the CF superposition never mixes $\Lambda$ levels) but must be taken into account if $\Lambda$-level mixing is needed.

[31] Edited by N. J. A. Sloane, The on-line encyclopedia of integer sequences: A100861 (2018), https://oeis.org.

[32] F. D. M. Haldane, Phys. Rev. Lett. 55, 2095 (1985).

[33] M. Fremling, J. Phys. A: Math. Theor. 46, 275302 (2013). 